\title{
Frontières
}

\section{Violences urbaines}

\section{De la fiction à la friction}

\section{Jean-Jacques Lavoie}

Volume 15, numéro 1, automne 2002

Délires urbains, dangers de mort

URI : https://id.erudit.org/iderudit/1073901ar

DOI : https://doi.org/10.7202/1073901ar

Aller au sommaire du numéro

\section{Éditeur(s)}

Université du Québec à Montréal

ISSN

1180-3479 (imprimé)

1916-0976 (numérique)

Découvrir la revue

Citer cet article

Lavoie, J.-J. (2002). Violences urbaines : de la fiction à la friction. Frontières, 15(1), 15-18. https://doi.org/10.7202/1073901ar

\section{Résumé de l'article}

Cet article propose quelques réflexions sur le rapport ville-violence mortifère à partir des premières pages de la Genèse et s'intéresse brièvement à l'histoire de leur réception dans les traditions juives et musulmanes, ainsi que dans la littérature occidentale.
Ce document est protégé par la loi sur le droit d'auteur. L'utilisation des services d’Érudit (y compris la reproduction) est assujettie à sa politique d'utilisation que vous pouvez consulter en ligne.

https://apropos.erudit.org/fr/usagers/politique-dutilisation/ 


\section{Résumé}

Cet article propose quelques réflexions sur le rapport ville-violence mortifère à partir des premières pages de la Genèse et s'intéresse brièvement à l'histoire de leur réception dans les traditions juives et musulmanes, ainsi que dans la littérature occidentale.

Mots clés : violence - mort - mythe

\section{Abstract}

This article presents a few thoughts on the deadly violence - city relationship, starting with the first pages of Genesis, and briefly concerns the history of the acceptance of these pages into Jewish and Muslim traditions as well as into Western literature.

Key words : violence - death - myth

\section{VIOLENCES \\ URBAINES \\ DE LA FICTION
À LA FRICTION \\ DE LA FICTION
À LA FRICTION}

UNE VILLE, UNE CAMPAGNE, DE LOIN C'EST UNE VILLE ET UNE CAMPAGNE, MAIS À MESURE QU'ON S'APPROCHE, CE SONT DES MAISONS, DES ARBRES, DES TUILES, DES FEUILLES, DES HERBES, DES FOURMIS, DES JAMBES DE FOURMIS, À L'INFINI. TOUT CELA S'ENVELOPPE SOUS LE NOM DE CAMPAGNE.

PASCAL, 1962, P. 65.

Jean-Jacques Lavoie, Ph.D.,

professeur, Département des sciences religieuses, UQÀM.

Plus que jamais, la ville se présente comme la fille du progrès et l'avenir du monde. «Arrive en ville » est le slogan préféré qui est adressé à ceux qu'on juge retardé et rétrograde. C'est pourquoi une bonne partie des gens des villes méprisent les ruraux, voire les banlieusards. Particulièrement depuis le $\mathrm{XX}^{\mathrm{e}}$ siècle, sur toute la planète, la population rurale a compris le message et ne cesse d'affluer vers les villes, dont la croissance galopante échappe de plus en plus à la maîtrise humaine. Les chiffres sont à ce sujet éloquents : «la population rurale s'est stabilisée à 0,5 pour cent durant ces 150 dernières années; dans le même temps, la population urbaine augmentait 5 fois plus vite » (Drewermann, 1993, p. 17). Il y a présentement environ 400 villes de plus d'un million d'habitants (Drewermann, 1993, p. 308) et les démographes estiment que la moitié de la population mondiale vit déjà en ville ${ }^{1}$.

Pourtant, au Nord comme au Sud et à l'Est comme à l'Ouest, de nombreuses mégavilles annoncent plutôt la mort de la société urbaine et rurale. Des cités comme São Paulo, Mexico, Le Caire, Lagos, Calcutta et Shanghai perturbent les équilibres écologiques, sociaux et économiques. Des agglomérations comme New York, Chicago, Los Angeles, Paris sont des amas de quar- tiers et de ghettos où maintes populations, souvent prisonnières et sans perspective d'avenir, sont condamnées à exprimer leur désespoir par des actes de violence.

Le XXI ${ }^{\mathrm{e}}$ siècle sera donc incontestablement celui des villes. Mais sera-t-il celui du progrès ou de la décadence meurtrière ? La réponse n'est pas facile, sans doute parce la question est formulée de façon trop dualiste. Pour les uns, l'histoire montre que le développement des villes a surtout été bénéfique à l'humanité, tandis que pour les autres elle aura plutôt été maléfique. Mon propos ici n'est pas de condamner la ville ni de faire l'éloge de la campagne. Je laisse cette tâche aux rousseauistes qui ont souvent été les premiers à bénéficier des avantages de la ville! Mon objectif est plus modeste. Il est même triplement limité. Premièrement, je compte simplement proposer quelques réflexions générales sur le rapport ville-violence mortifère. Deuxièmement, ces réflexions se feront surtout à partir des premiers chapitres de la Genèse, particulièrement le chapitre 4 qui raconte le meurtre d'Abel par son frère Caïn. Troisièmement, je ne m'attarderai qu'à quelques aspects de ces pages qui nous proposent une riche et complexe anthropologie religieuse, car ce que Pascal dit de la ville, de la campagne, il faut aussi le dire des mots, des textes: les premières pages de la Bible recèlent donc des sens, des signifiés à partir desquels on pourrait méditer à l'infini. 


\section{LA VILLE, FILLE DU PROGRÈS OU FILLE D'UN MEURTRIER ?}

L'histoire des idées nous apprend que des jugements opposés sur la ville ont toujours existé. Les êtres humains ont toujours rêvé de la ville idéale en la plaçant au centre de leur utopie, voire de leur eschatologie. On sait que La Mecque, par son patronyme même (Omm al-Qourra, "Mère des cités »), est l'Omphalos du Monde ou Prototype des Mondes (Coran 6,92 ; 28,59 ; 42,7 ; 46,27), elle est le symbole de la présence divine sur terre. C'est aussi une ville, Jérusalem, qui contiendra tous ceux que Dieu choisira à la fin des temps (Apocalypse 21). Il en va de même pour la tradition juive : la reconstruction de Jérusalem est un des plus grands symboles du messianisme juif et il est écrit dans le Talmud que Dieu fit le serment de ne pas pénétrer dans la Jérusalem céleste avant d'être entré dans la Jérusalem terrestre reconstruite (Ta'anit 5a).

Par ailleurs, si l'on se réfère à certains textes anciens, il semble que ville et mort aient constitué un couple dès l'origine. Ainsi, après avoir décidé de fonder une ville, Romulus tue son jumeau Remus. Comme si l'édification d'une ville exigeait le prix d'un fratricide. Lorsque la ville est fondée, la population locale étant insuffisante, Romulus réserve une partie de la ville à tous les fugitifs et hors-la-loi du pays. Tel est le noyau de la première population de Rome. Mais comme il n'y a que des hommes, Romulus imagine un stratagème pour dérober les femmes de ses voisins, les Sabines. La réussite du stratagème déclenche la guerre et provoque de nombreux morts (voir, par exemple, Plutarque, 9,119,10). La culture citadine semble donc responsable de la croissance vertigineuse des conflits et de la violence meurtrière.

À ce sujet, le chapitre 4 de la Genèse est également éloquent: Caïn, l'homme qui a inventé la mort, est aussi celui qui a créé la première ville. Entre la faute d'Adam et Ève et le meurtre d'Abel, personne n'était encore mort. La menace de Dieu n'était que virtuelle. Toutefois, dès que l'humanité tient son histoire en main, ce qu'elle crée immédiatement, c'est la mort, puis une ville. Abel est le premier mort de l'histoire de l'humanité et sa mort est un fratricide. Aussitôt après avoir commis son meurtre, Caïn est privé des fruits du sol et condamné par Dieu à devenir errant et vagabond (nâd) sur la terre (Genèse 4,12). Que fait-il ? Il se plaint, s'éloigne de la présence de Dieu pour habiter le pays de Nod, c'est-à-dire le pays de l'errance ${ }^{2}$ (Genèse 4,13-16), puis il $\mathrm{y}$ construit la première ville (Genèse 4,17 ) ! Dans sa relecture de Genèse 4, 17, Flavius Josèphe, historien juif du premier siècle de notre ère, apporte quelques précisions : « Il (Caïn) fut le premier à établir un cadastre de la terre et à construire une ville; il la fortifia par des murailles et obligea ses habitants à s'assembler dans un même lieu » (Antiquités juives I, 62).

Ainsi, non seulement Caïn n'erre pas toute sa vie, mais le récit de Genèse 4 comme celui de Flavius Josèphe indiquent que son errance est de très courte durée. Construire une ville, c'est bien ce qu'il y a de plus opposé à l'errance, puisque c'est d'abord construire ses remparts, symbole de sécurité et de pouvoir. Construire une ville est une façon de conjurer non seulement l'errance, mais aussi et surtout la mort. C'est ce que reconnaissent aussi le Midrash Bereshith Rabbah 23,1 et le traité Tehillim 9,85 du Talmud de Babylone qui spécifient que Caïn s'efforce d'immortaliser son nom grâce à des monuments et que c'est ainsi qu'il devient bâtisseur de cités. "La ville, comme la pierre tombale, et plus encore, en raison de son ampleur, proclame la nonmort »(Thomas, 1979, p. 192).

À cette première ville, Caïn donne le nom de son premier fils: Hénok, mot qui contient l'idée d'inauguration, de dédicace, de fondation, de commencement ${ }^{3}$. Ce nom laisse entendre que la construction de la ville constitue un geste sacré. Or, en sachant que la ville est originellement une copie de la cosmogonie et de l'univers (Keel, 1997, p. 118-120), il est permis de croire que le geste de Caïn vise à imiter la création divine, non par piété mais bien par nostalgie. Chassé de la création divine, Caïn s'efforce de se reconstruire un paradis dans le pays de l'exil et ce paradis a pour nom «ville ${ }^{4}$.

Récapitulons : condamné à l'errance parce qu'il a tué son frère, Caïn crée la première ville et lui donne le nom d'Hénok, nom qui laisse entendre que la ville se présente comme un substitut de l'Éden à jamais perdu. Et Dieu n'intervient pas. Pire: l'enchaînement des meurtres que Dieu voulait éviter reprend de plus belle (Genèse 4,2324). C'est ainsi que les villes deviennent rapidement les lieux de toutes les violences.

Babel, dont le nom signifie "porte du dieu », est la première nommée dans la série des grandes villes d'empires qui se distingueront par leur violence (Genèse 11,4). La tradition juive nous fournit encore des détails sur la manière inhumaine et industrielle dont cette ville et sa tour sont édifiées. La construction est si longue et laborieuse qu'une brique devient plus précieuse aux yeux des constructeurs qu'un être humain. Si un ouvrier trouve la mort sur le chantier de construction, personne n'y prête attention, car ils sont des milliers à y travailler. Par contre, si une brique se brise, les constructeurs se lamentent (voir, par exemple, Pirqé Rabbi Éliézer 48). Ils sont tellement zélés dans l'accomplissement de leur tâche qu'ils ne permettent pas à une femme d'arrêter la confection des briques même à l'heure de l'enfantement (3 Baruch 3,5). En résumé, ni la naissance ni la mort ne préoccupent ces premiers industriels. Puis, l'on connaît le défi qu'ils tentent ainsi de relever : construire leur unité par l'uniformité et donc nier toute forme d'altérité. Là, Dieu brise aussitôt l'impérialisme destructeur (Genèse 11,1-9). Quant aux habitants des villes de Sodome et Gomorrhe, ils sont les premiers à violer le devoir d'hospitalité et ils en subissent le châtiment (Genèse 19). Bref, la liste des passages où les citadins ne jouissent pas d'une bonne réputation pourrait facilement s'allonger.

C'est peut-être pourquoi l'historien, théologien et juriste musulman Tabarî, (225/839-310/923), dans sa conclusion du récit de Caïn, propose un verdict sans

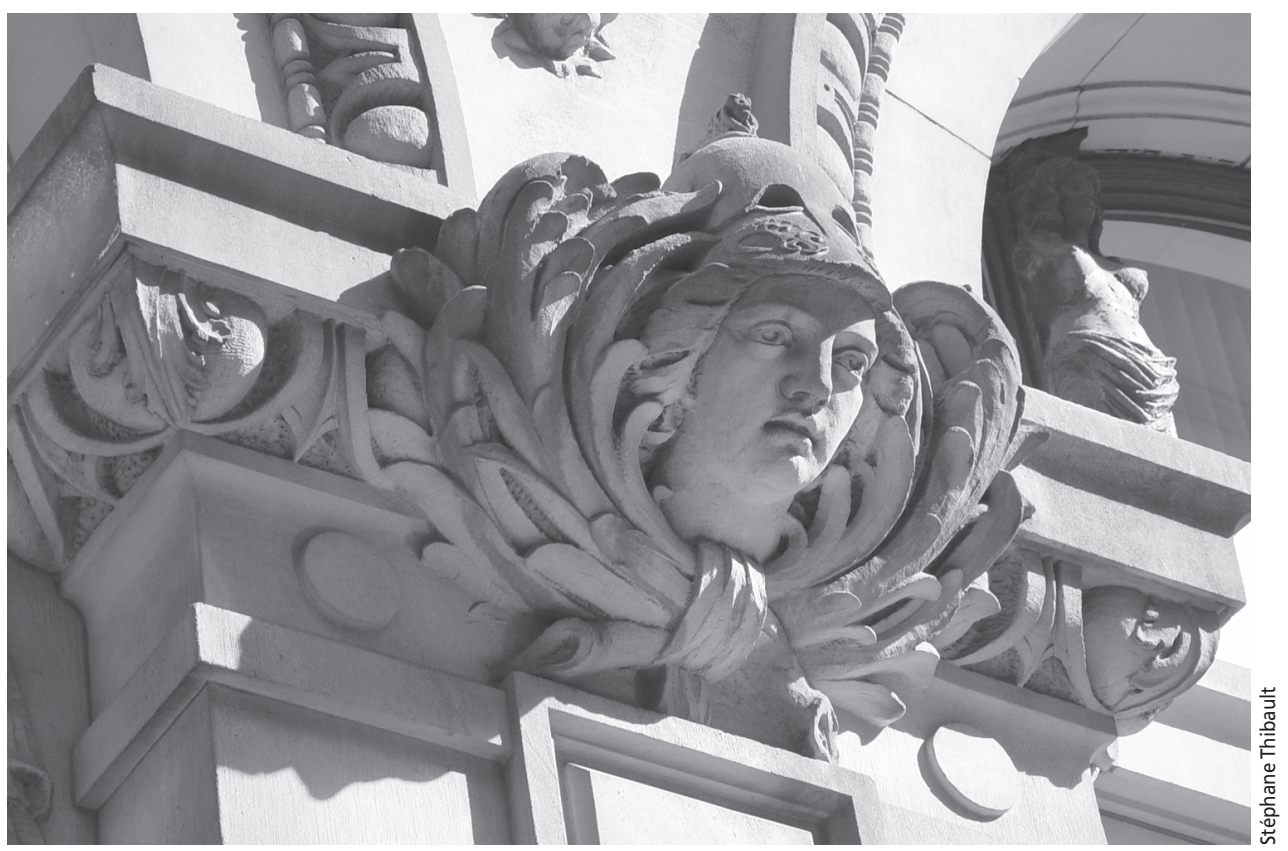




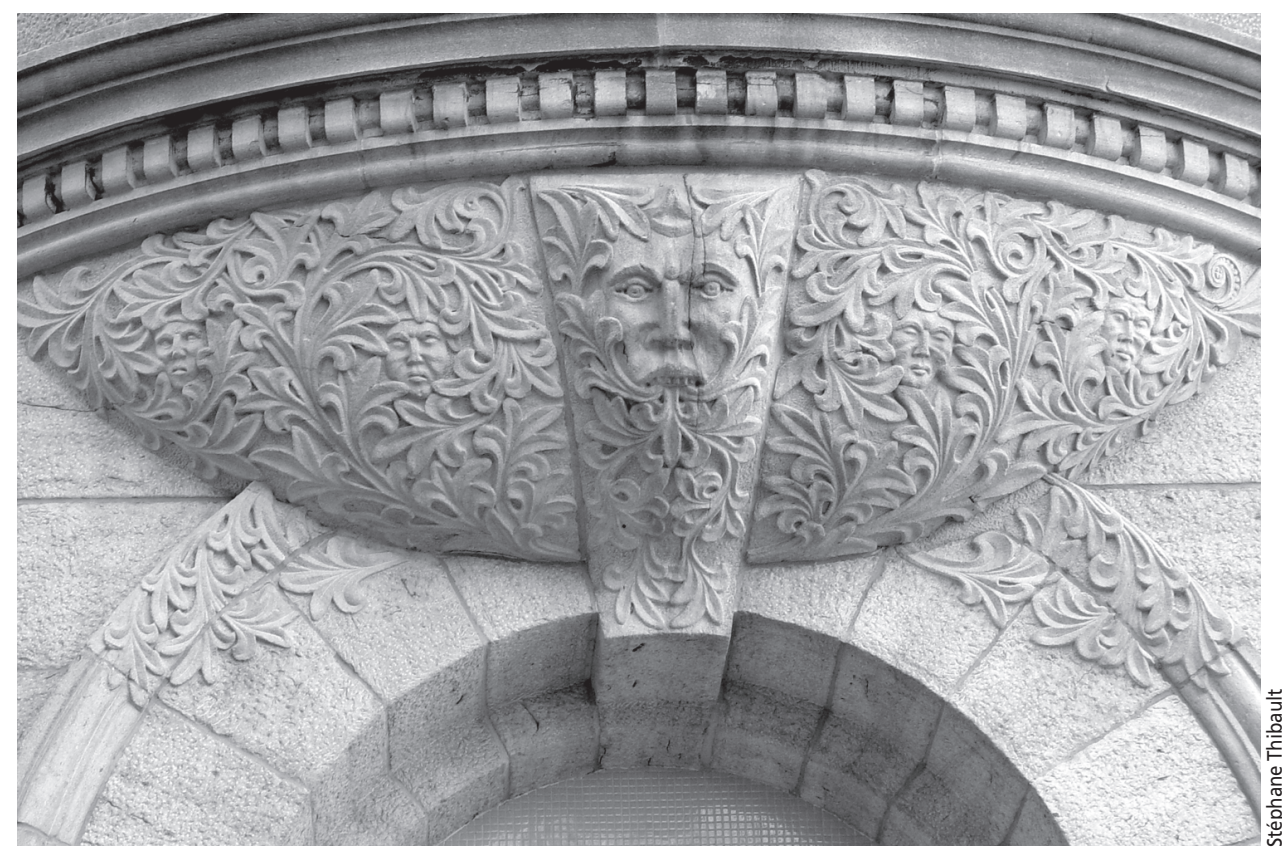

nuance. Voici le début du discours conclusif qu'il fait dire à Adam : «Les villes présentent toutes le même aspect; les hommes sont corrompus. La face de la terre est devenue un désert salé ; elle est devenue hideuse » (Tabarî, 2001, p. 88).

\section{DU MYTHE À L'HISTOIRE}

Bien entendu, la genèse de la vie urbaine ne saurait être ramenée à une histoire aussi courte! Que penser alors de ces premières pages de la Genèse? Doivent-elles nous inciter à condamner sans appel la vie urbaine ? Ce serait là une attitude réactionnaire. C'est pourtant le comportement qu'ont eu les Rékabites, un groupe de Juifs, qui, encore au $\mathrm{VI}^{\mathrm{e}}$ siècle avant notre ère, refusaient toujours toute forme de vie sédentaire et citadine (Jérémie 35). Plus près de nous, c'est aussi ce qu'ont fait les Khmers rouges transformant brutalement la ville de Phnom Penh en désert, mais au nom d'une autre idéologie : le communisme ! Condamner sans appel la vie urbaine serait aussi une option aveugle ; l'espérance de voir apparaître une nouvelle Jérusalem à l'horizon eschatologique ne traverse-t-elle pas non seulement la Bible et le Talmud, comme on l'a entrevu ci-haut, mais aussi toute l'histoire juive et chrétienne? (À ce sujet, voir, par exemple, Delumeau, 2000, p. 92-107.)

Doit-on voir dans ces premiers chapitres de la Genèse un simple mythe? Certes, le texte de Genèse 1-11 est bel et bien un récit mythique, mais c'est précisément pourquoi il est si significatif et toujours d'actualité ! Force est de constater que les fils de Caïn existent toujours et que l'indifférence des constructeurs de la ville de Babel a préfiguré celle des bâtisseurs de l'ère industrielle. En d'autres mots, toute l'histoire de l'humanité a été violente et non pas seulement celle de
l'Antiquité. De nos jours, la violence est toujours à l'œuvre, particulièrement dans les milieux urbains, avec toutefois une différence : les moyens ne cessent de devenir plus terrifiants. Les chercheurs qui ont étudié les violences urbaines ${ }^{5}$ sont unanimes pour dire, avec des statistiques troublantes à l'appui, qu'elles ne cessent de s'aggraver depuis les dernières décennies (van Grasdorff et Séroussi, 1996, p. 34 ; Bousquet, 1998, p. 3843 ; Garnier, 1999, p. 104 ; Bauer et Raufer, 2000, p. 7-46). Quelques exemples suffiront à illustrer ce triste propos. Des études ont montré que l'indice de criminalité croît avec la hauteur des bâtiments (voir déjà la tour de Babel!) et avec la taille des agglomérations (Thomas, 1979, p. 184-185) ${ }^{6}$. Le taux de criminalité est si élevé que la police - mot qui dérive justement du grec polis, "cité », et qui montre bien que la ville n'est pas en soi un lieu sécuritaire - ne suffit plus à assurer la quiétude des citadins. C'est pourquoi ceux-ci, comme le Caïn hugolien traqué par l'œil attentif de Dieu (Hugo, 1950, p. 25-26), évoluent de plus en plus sous le regard omniprésent des caméras de vidéosurveillance.

En outre, il n'y a pas que les noms proqui sont significatifs. Les noms communs donnés aux villes ou à des quartiers de métropole sont eux aussi révélateurs de la misère et de la violence meurtrière que subissent des populations de plus en plus nombreuses. Des termes comme bidonville, ghetto, slum, favela, chabalos, barrio de las latas, etc., apparaissent comme autant de noms de maladies qui gangrènent nos villes. Ces quelques exemples illustrent bien comment les métropoles, terme qui dérive des mots grecs «mère » et "cité », sont loin d'être des mères pour leurs habitants. pres donnés aux premières villes de la Bible

\section{LA VILLE, UN LIVRE OUVERT}

La ville est une écriture; celui qui se déplace dans la ville, c'est-à-dire l'usager de la ville (ce que nous sommes tous), est une sorte de lecteur qui, selon ses obligations et ses déplacements, prélève des fragments de l'énoncé pour les actualiser en secret. Quand nous nous déplaçons dans une ville, nous sommes tous dans la situation du lecteur des 100000 millions de poèmes de Queneau, où l'on peut trouver un poème différent en changeant un seul vers; à notre insu, nous sommes un peu ce lecteur d'avant-garde lorsque nous sommes dans une ville.

\section{(Barthes, 1985, p. 268)}

Puisque la ville est un livre ouvert et qu'une ville aussi bien qu'un texte recèle des sens et des signifiés inépuisables, le procès intenté à la ville pourrait se poursuivre indéfiniment. Cependant, il ne saurait être complet et équitable s'il ne débouche pas sur une interrogation de la société et des individus qui la composent. La violence meurtrière qui règne dans les milieux urbains plus que partout ailleurs ne relèvet-elle pas d'une crise démographique, sociale et économique? Certes, mais selon le récit de Genèse 4 , cette violence relève d'une crise religieuse. En effet, Caïn incarne dans ce passage l'être humain primordial, paradigmatique, au même titre qu'Adam et Ève dans les chapitres antérieurs. Toutefois, si Adam et Ève représentaient les êtres humains devant Dieu, Caïn est la figure de l'être humain devant son semblable. Et c'est parce que l'être humain a nié la différence qui existe entre lui et Dieu qu'il ne veut pas faire droit à la différence qui existe entre lui et ses semblables. Au mensonge de l'être humain devant Dieu (Genèse 3,12-13) fait place la violence de l'être humain face à son semblable. La question "Où es-tu? » posée à Adam (Genèse 3,9) fait dès lors place au «Où est ton frère? » (Genèse 4,9) adressé à Caïn. On connaît sa réponse : "Suis-je le gardien de mon frère ? " (Genèse 4,9.) L'actualité de cette interrogation ne tient-elle pas au fait qu'elle aurait été écrite pour des citadins qui avaient perdu le sens de la fraternité?

\section{BIBLIOGRAPHIE}

BARTHES, R. (1985). L'aventure sémiologique, Paris, Seuil.

«Baruch (Apocalypse grecque de)» (1987). Texte traduit, présenté et annoté par J. Hadot, dans A. Dupont-Sommer et al. (dir.), La Bible. Écrits intertestamentaires, Paris, Gallimard. 
BAUER, A. et X. RAUFER (2000). Violences et insécurité urbaines, Paris, Presses universitaires de France.

BOUSQUET, R. (1998). Insécurité: nouveaux risques. Les quartiers de tous les dangers, Paris et Montréal, L'Harmattan.

BRUYELLE, P. (2000). Les très grandes concentrations urbaines, Liège, Sedes.

CHEVALIER, J. (2000). «"Violences urbaines" et tentations de sécession sociale », dans Th. Ferenczi (dir.), Faut-il s'accommoder de la violence?, Bruxelles, Éditions complexe, p. 163-177.

DELUMEAU, J. (2000). Que reste-t-il du paradis ?, Paris, Fayard.

DREWERMANN, E. (1993). Le progrès meurtrier, Paris, Stock.

GARNIER, J.P. (1999). Le nouvel ordre local. Gouverner la violence, Paris, L'Harmattan.

HUGO, V. (1950). La légende des siècles. La fin de Satan. Dieu, Paris, Gallimard.

JOSÈPHE, F. (1929). "Antiquités juives", dans Euvres complètes de Flavius Josèphe traduites en français sous la direction de Th. Reinach, Paris, Leroux.

KEEL, O. (1997). The Symbolism of the Biblical World. Ancient Near Eastern Iconography and the Book of Psalms, traduit par T.J. Hallet, Winona Lake, Eisenbrauns.

LECONTE DE LISLE (1974). Poésies complètes. Tome II. Poèmes barbares, Genève, Slatkine.
Midrach Rabba (1951). Traduit sous la direction de H. Freedman et M. Simon, Londres, Soncino.

PASCAL, B. (1962). Pensées, Paris, Seuil.

Piqé de Rabbi Éliézer (Leçon de Rabbi Éliézer) (1983). Traduit et annoté par M.-A. Ouaknin et É. Smilévitch, Paris, Verdier.

PLUTARQUE (1964). Vies, Tome I, texte établi et traduit par R. Flacelière et al., Paris, Les Belles-Lettres.

TABARÎ (2001). La Chronique. Histoire des prophètes et des rois. De la création à David. De Salomon à la chute des Sassanides, traduit du persan par H. Zotenberg, Paris, Actes Sud/Sinbad.

Talmud de Babylone (1935-1958). Traduit sous la direction de I. Epstein, Londres, Soncino.

THOMAS, L.-V. (1979). Civilisation et divagations. Mort, fantasmes, sciencefiction, Paris, Payot.

VAN GRASDORFF, G. et R. SÉROUSSI (1996). La violence. État des lieux, Paris, France-Empire.

\section{Notes}

1. Le lecteur intéressé à lire des colonnes entières de chiffres pourra toujours consulter le collectif dirigé par Pierre Bruyelle (2000) ainsi que le site de l'Institut national d'études démographiques à l'adresse suivante: http://www.ined.fr
2. Le pays de Nod est inconnu parce que la toponymie a ici une signification strictement symbolique: il s'agit d'un jeu de mots entre $n a d$, "vagabond» (Genèse 4,12-13) et nod, « errance» (Genèse 4,16).

3. Le mot dérive du verbe kânak, «commencer», « inaugurer », « initier ».

4. Envoûtés par un autre mythe, celui du Progrès, les écrivains romantiques du $\mathrm{XIX}^{\mathrm{e}}$ siècle réhabiliteront non seulement ce mythe d'une ville-paradis du nom d'Hénokhia, mais aussi son fondateur Caïn, lequel sera confondu avec Prométhée. La version la plus complète de ce nouveau mythe de la cité d'Hénokhia se retrouve chez Leconte de Lisle, dans un long poème paru en 1869 et qui s'inspire de Byron et Lamartine (Leconte de Lisle, 1974, p. 1-21).

5. L'expression «violences urbaines» montre bien à elle seule comment il est malaisé de dire les origines de la violence, de désigner distinctement le ou les groupes qui revendiquent le recours à la violence. Cette expression euphémise non seulement les actes et les protagonistes (malfaiteurs et victimes), mais aussi la géographie de la violence. Voir à ce sujet Chevalier, 2000, p. 163-177.

6. Toujours avec des exemples à l'appui, Thomas insiste aussi sur le fait que la ville, qui est le milieu social où le taux de mortalité criminelle est proportionnellement le plus élevé, est aussi, fort paradoxalement, le lieu où le déni de la mort est le plus manifeste (Thomas, 1979, p. 187-188).
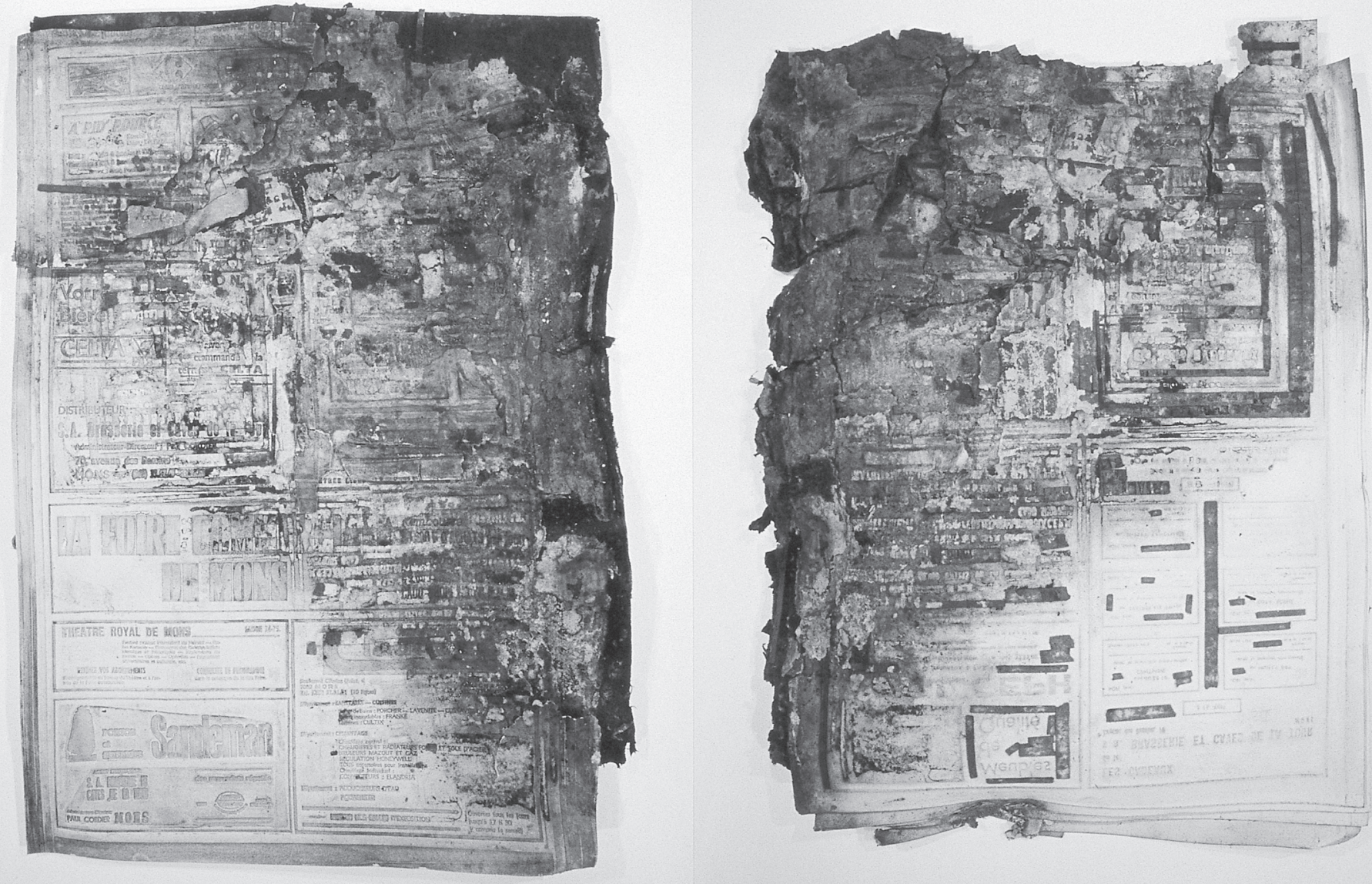